\title{
Effect of Ca doping on thermally activated flux flow in the $\mathrm{Y}_{3} \mathrm{Ba}_{5} \mathrm{Cu}_{8} \mathrm{O}_{18}$ superconductor
}

\author{
MUSTAFA AKYOL ${ }^{1}$, ALI OSMAN AYAŞ ${ }^{2}$, GÖNÜL AKÇA $^{1}$, SELDA KILIÇ ÇETIN ${ }^{1}$ and \\ AHMET EKİCIBíL ${ }^{1, *}$ \\ ${ }^{1}$ Department of Physics, Faculty of Sciences and Letters, Çukurova University, 01330 Adana, Turkey \\ ${ }^{2}$ Department of Physics, Faculty of Sciences and Letters, Adıyaman University, 02040 Adiyaman, Turkey
}

MS received 22 January 2015; accepted 7 April 2015

\begin{abstract}
Y}_{3} \mathrm{Ba}_{5} \mathrm{Cu}_{8} \mathrm{O}_{18}$ (Y-358) and $\mathrm{Y}_{3} \mathrm{Ba}_{5} \mathrm{Ca}_{2} \mathrm{Cu}_{8} \mathrm{O}_{18}$ (YCa-358) compounds were prepared by the so-called sol-gel method. The effect of doping $\mathrm{Ca}$ atoms into the $\mathrm{Y}_{3} \mathrm{Ba}_{5} \mathrm{Cu}_{8} \mathrm{O}_{18}$ superconductor is studied by employing the electrical resistivity measurements at various magnetic fields. The possible degradation in microstructural and superconducting properties due to the addition of $\mathrm{Ca}$ was discussed. The resistivity as a function of temperature measurements shows that the depression in superconducting temperature is more pronounced for both samples. A systematic analysis of the magnetoresistivity of the Y-358 and YCa-358 compounds has been carried out by using the thermally activated flux flow (TAFF) model. The TAFF activation energy, $U$, is field dependent and obeys the power law $U=c H^{-\alpha}$, where $\alpha$ increases while $c$ decreases with the addition of Ca. Furthermore, $U$ and the calculated upper critical field, $H_{\mathrm{c} 2}$, decrease with the addition of $\mathrm{Ca}$.
\end{abstract}

Keywords. Y-based superconductor; magnetoresistivity; activation energy; coherence length.

\section{Introduction}

Y-based (YBCO) superconductors attract great technological interest, as their superconducting transition temperatures, $T_{\mathrm{c}}$, exceed the liquid-nitrogen temperature. In the YBCO system, the electrical transport properties ${ }^{1}$ are drastically changed by the content of oxygen deficiency and the ordering of oxygen ions in the $\mathrm{CuO}$ chain sites which affects the doping level, ${ }^{2}$ the conductivity mechanism and the property of the spin system. ${ }^{3}$ The $\mathrm{YBa}_{2} \mathrm{Cu}_{3} \mathrm{O}_{7-\delta}$ (Y-123) has superconductivity transition temperature between $T_{\mathrm{c}} 80$ and $93 \mathrm{~K},{ }^{4}$ and many attempts have been made to find new forms of the material offering higher $T_{\mathrm{c}}$ and better superconducting properties. A great deal of studies have been carried out to obtain new compositions of the YBCO superconductors, like $\mathrm{YBa}_{2} \mathrm{Cu}_{4} \mathrm{O}_{8}(\mathrm{Y}-124)^{5}$ and $\mathrm{Y}_{2} \mathrm{Ba}_{4} \mathrm{Cu}_{7} \mathrm{O}_{15}(\mathrm{Y}-247){ }^{6}$ which are different from the point of the numbers of their $\mathrm{CuO}_{2}$ planes and $\mathrm{CuO}$ chains or double chains and their relative positions. $\mathrm{Y}_{3} \mathrm{Ba}_{5} \mathrm{Cu}_{8} \mathrm{O}_{18}(\mathrm{Y}-358)$ has been in recent times synthesized with a transition temperature of about $2 \mathrm{~K}$ higher than that of $\mathrm{Y}-123 .^{7-9}$ This compound consists of five $\mathrm{CuO}_{2}$ layers and three $\mathrm{CuO}$ chains in the unit cell.

Among the many remarkable mixed state properties of the high-temperature superconductors, the resistivity has attracted much attention. ${ }^{10,11}$ Resistivity measurements

*Author for correspondence (ahmetcan@cu.edu.tr) under magnetic fields yield important information about the flux motions in the superconductors. For a finite current density, the Lorentz force is sufficiently strong so that the force on vortex lines in the vortex glass region can overcome a certain class of pinning barriers, leading to a flux creep, and a finite voltage.

The flux line dynamics can be divided into three regimes (i) flux flow, $J>J_{\mathrm{c}}$; (ii) thermally activated flux flow (TAFF), $J \ll<J_{\mathrm{c}}$ and (iii) flux creep, $J \sim J_{\mathrm{c}}$ in the transition region between $T_{\text {c.on }}$ and $T_{\text {c.off }}$. Flux flow and flux creep are common terms for low-temperature superconductors, but TAFF has been coined for a new aspect of high-temperature superconductors. Flux flow phenomena arise when the current and the applied magnetic fields in a superconductor are high enough to essentially tear loose of the flux lattice and thus to set the flux in sliding motion. In high-temperature superconductors, it has been observed that even for very low current densities, $J \ll J_{c}$, superconductors under high enough magnetic fields may display an exponential decay of resistivity with decreasing temperature well below $T_{\text {c.on. }}$. This behaviour is due to the phenomenon known as TAFF as suggested by Hughes. ${ }^{12}$ Thus, the TAFF is closely associated with the flux current density limit.

Therefore, in the TAFF region the resistivity is still nonzero and its temperature dependence follows an Arrhenius law of the form ${ }^{13}$

$$
\rho(T, H)=\rho_{0} \exp \left(-U_{0}(T, H) / k_{\mathrm{B}} T\right) .
$$


There have been many reports regarding temperature and field dependence of the activation energy in various hightemperature superconductors. Palstra et $a l^{13}$ reported the power law dependence of $U$ under magnetic field, $U \sim H^{-\alpha}$, whereas Kucera et $a l^{14}$ suggested that $U \sim H^{-1 / 2}\left(1-T / T_{\mathrm{c}}\right)$, where $T_{\mathrm{c}}$ is the critical temperature. The same relation was suggested by Wagner et al. ${ }^{15}$ In a recent report by Zhang et al, ${ }^{16}$ an empirical form as $U \sim H^{\alpha}(1-t)^{\beta(H)}$ was suggested.

In this work, we report the effect of the addition of $\mathrm{Ca}$ to the $\mathrm{Y}_{3} \mathrm{Ba}_{5} \mathrm{Cu}_{8} \mathrm{O}_{18}(\mathrm{Y}-358)$ compound, $\mathrm{Y}_{3} \mathrm{Ba}_{5} \mathrm{Ca}_{2} \mathrm{Cu}_{8} \mathrm{O}_{18}$ (YCa-358), from the point of the activation energy and flux flow mechanisms using the transport data of the compounds under magnetic field.

\section{Experimental}

$\mathrm{Y}_{3} \mathrm{Ba}_{5} \mathrm{Cu}_{8} \mathrm{O}_{18}(\mathrm{Y}-358)$ and $\mathrm{Y}_{3} \mathrm{Ba}_{5} \mathrm{Ca}_{2} \mathrm{Cu}_{8} \mathrm{O}_{18}$ ( $\left.\mathrm{YCa}-358\right)$ compounds have been prepared by the sol-gel method and these will be labelled as $\mathrm{Y}-358$ and $\mathrm{YCa}-358$, respectively. Appropriate amounts of $\mathrm{Y}_{2} \mathrm{O}_{3}, \mathrm{Ba}\left(\mathrm{NO}_{3}\right)_{2}, \mathrm{CuO}$ and $\mathrm{CaCO}_{3}$ were first dissolved in dilute $\mathrm{HNO}_{3}$ solution at $150^{\circ} \mathrm{C}$ and then citric acid and ethylene glycol were added to the mixture. Afterwards residual material was dried slowly at $300^{\circ} \mathrm{C}$ until a dry-gel was formed. Finally, the residual precursor was burned in air at $600^{\circ} \mathrm{C}$. The materials thus obtained were first ground by using an agate mortar to have a fine powdered form and then the pellets with $13 \mathrm{~mm}$ radius and $2 \mathrm{~mm}$ thicknesses were produced from each powdered composition by pressing them under a pressure of $3 \mathrm{t}$. The pellets were then separately sintered at 890 and $900^{\circ} \mathrm{C}$ for $72 \mathrm{~h}$ in air, respectively, and then cooled down to the room temperature in the furnace. The resistivity measurements of the compounds, under magnetic fields up to $2 \mathrm{~T}$, were carried out by employing the standard four-probe method with silver paint contacts. For measurements at low temperatures from 50 to $100 \mathrm{~K}$ under magnetic fields up to $2 \mathrm{~T}$, we have used a Quantum Design PPMS with a closed cycle helium cryostat.

\section{Results and discussion}

The resistivity $v s$. temperature plots for the samples of Y358 and YCa-358 under magnetic fields of $0.0,0.1,0.5$, 1.0 and $2.0 \mathrm{~T}$ are shown in figure $1 \mathrm{a}$ and $\mathrm{b}$, respectively. The trends of the curves for both of the compounds are more or less the same with the increase in the applied fields. It has been found that the resistivity values of the samples in their normal states decrease almost linearly with the decrease in temperature, indicating a metallic behaviour, and then sharply drop to lower values leading to transitions to the superconducting states of them. Table 1 shows the superconducting transition temperatures associated with the onset, $T_{\text {c.on }}$, and offset, $T_{\text {c.off }}$, of the
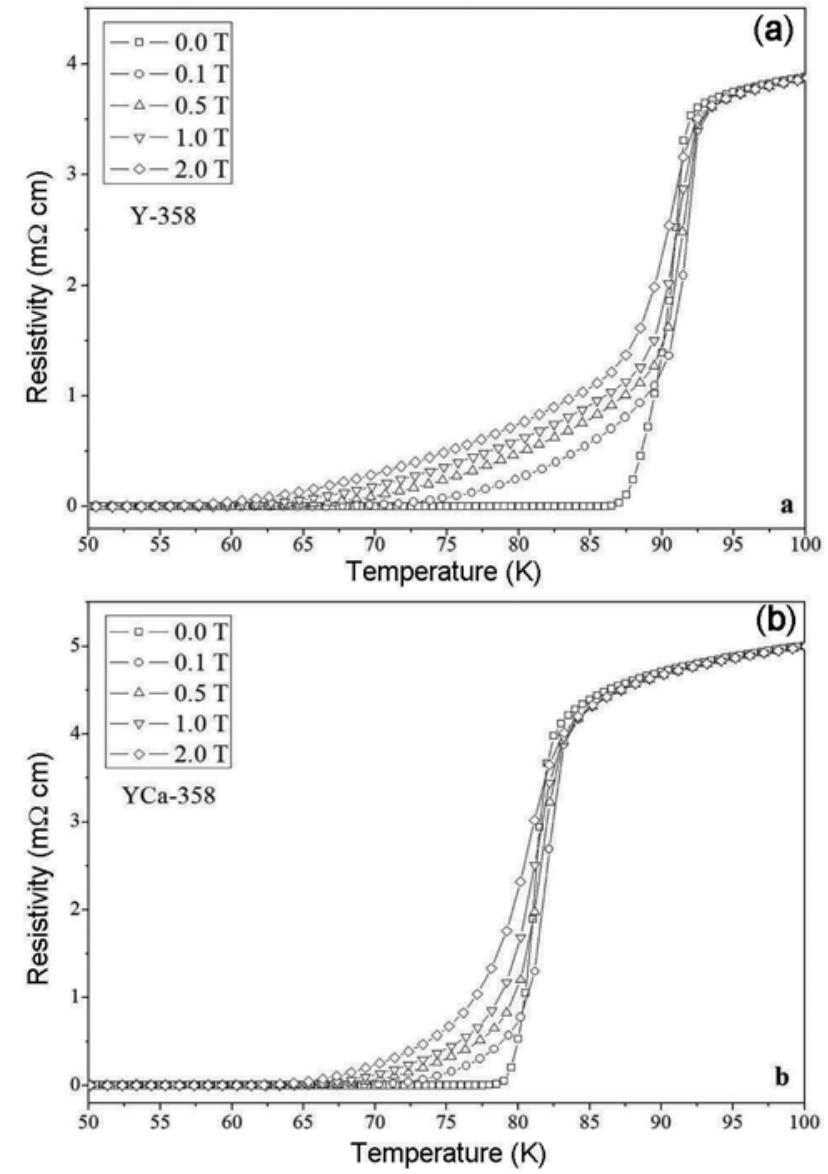

Figure 1. The influence of applied magnetic fields on the resistivity $v s$. temperature plots for the compounds: (a) $\mathrm{Y}_{3} \mathrm{Ba}_{5} \mathrm{Cu}_{8} \mathrm{O}_{18}$ and (b) $\mathrm{Y}_{3} \mathrm{Ba}_{5} \mathrm{Ca}_{2} \mathrm{Cu}_{8} \mathrm{O}_{18}$.

compounds under the above-mentioned applied magnetic fields. As it can be seen from table $1, T_{\text {c.on }}$ values for the compound $\mathrm{YCa}-358$ are considerably lower than that of the main compound Y-358, at the corresponding magnetic fields, however, it is the reverse for the $T_{\text {c.off }}$ values, especially at high fields, and as expected $T_{\text {c.on }}$ and $T_{\text {c.off }}$ values decrease with the increase in the applied magnetic field. In particular, the $T_{\text {c.off }}$ values quickly shift to lower temperatures. This behaviour can be explained as following: it is well known that in type-II superconductors, an electric field arises, and hence an electrical resistivity and energy dissipation occurs when the driving Lorentz force is increased with the increase in the magnetic field per unit volume. As lowering the temperature increases the pinning forces at lower temperatures, higher magnetic fields are needed for the depinning the flux lines. These are the reasons why $T_{\text {c.off }}$ shifts to the lower temperatures with the increase in the applied magnetic fields. As a result, the application of higher magnetic fields causes a shift of the zero resistivity temperatures downward to the lower temperatures. Table 1 also includes $\Delta T=T_{\text {c.on }}-$ $T_{\text {c.off }}$ values which, as expected, increase with the increase in the magnetic field. Table 1 clearly shows that 
Table 1. The magnetic field dependence of transition temperatures of the compounds $\mathrm{Y}_{3} \mathrm{Ba}_{5} \mathrm{Cu}_{8} \mathrm{O}_{18}$ and $\mathrm{Y}_{3} \mathrm{Ba}_{5} \mathrm{Ca}_{2} \mathrm{Cu}_{8} \mathrm{O}_{18}$.

\begin{tabular}{|c|c|c|c|c|c|}
\hline \multirow[b]{2}{*}{ Sample code } & \multicolumn{5}{|c|}{ Transition temperatures $(\mathrm{K})$} \\
\hline & $0 \mathrm{~T}$ & $0.1 \mathrm{~T}$ & $0.5 \mathrm{~T}$ & $1.0 \mathrm{~T}$ & $2.0 \mathrm{~T}$ \\
\hline Y-358 & $\begin{array}{c}T_{\text {c.on }}=92.7 \\
T_{\text {c.off }}=87.6 \\
\Delta T=5.11\end{array}$ & $\begin{aligned} T_{\text {c.on }} & =91.3 \\
T_{\text {c.off }} & =72.2 \\
\Delta T & =19.1\end{aligned}$ & $\begin{aligned} T_{\text {c.on }} & =90.24 \\
T_{\text {c.off }} & =65.1 \\
\Delta T & =25.14\end{aligned}$ & $\begin{aligned} T_{\text {c.on }} & =90.24 \\
\text { c.off } & =62.3 \\
\Delta T & =27.94\end{aligned}$ & $\begin{aligned} T_{\text {c.on }} & =89.24 \\
T_{\text {c.off }} & =59.7 \\
\Delta T & =29.54\end{aligned}$ \\
\hline YCa-358 & $\begin{aligned} T_{\text {c.on }} & =86.5 \\
T_{\text {c.off }} & =79.4 \\
\Delta T & =7.10\end{aligned}$ & $\begin{aligned} T_{\text {c.on }} & =85.6 \\
T_{\text {c.off }} & =71.1 \\
\Delta T & =14.5\end{aligned}$ & $\begin{aligned} T_{\text {c.on }} & =85.0 \\
T_{\text {c.off }} & =67.3 \\
\Delta T & =17.7\end{aligned}$ & $\begin{aligned} T_{\text {c.on }} & =84.7 \\
T_{\text {c.off }} & =65.5 \\
\Delta T & =19.2\end{aligned}$ & $\begin{aligned} T_{\text {c.on }} & =84.2 \\
T_{\text {c.off }} & =63.0 \\
\Delta T & =21.2\end{aligned}$ \\
\hline
\end{tabular}

the $\Delta T$ values of $\mathrm{Y}-358$ obtained in magnetic fields decrease with the addition of $\mathrm{Ca}$.

The data of magneto-resistive transition can be used to determine the temperature dependence of the irreversibility field. Here, we define the zero resistivity transition temperature under the application of magnetic fields as the temperature $T_{\text {irr }}\left(T_{\text {c.off }}\right)$ and call the corresponding magnetic field at the irreversibility field $B_{\text {irr. }}$ The plots in figure 2 show the irreversibility magnetic field values $v s$. the onset and offset temperatures, for the compounds Y358 and YCa-358, deduced from the magnetoresistivity data given in figure $1 \mathrm{a}$ and $\mathrm{b}$. It is obvious that within the studied magnetic field range, while the amounts of variations of the onset temperatures are only about $5 \mathrm{~K}$, they are about 27 and $17 \mathrm{~K}$ for the offset temperatures of $\mathrm{Y}-358$ and $\mathrm{YCa}-358$, respectively. All these results strongly suggest that the superconductivity can completely diminish with a high enough magnetic field, as expected. Certainly, apart from the effect of strong magnetic fields the formation of weak coupling between the superconducting grains and the intergrowth of the impurity solid solution phase within the superconducting regions also play a crucial role in diminishing the superconductivity. However, an optimum applied magnetic field can hinder the negative effects arising from the impurities on the superconductivity.

The study of TAFF is manifested as a broadening of the superconducting transition. In the presence of an applied magnetic field, such broadening is interpreted in terms of energy dissipation caused by vortex motion. The resistivity in the TAFF region is caused by the TAFF of the vortices. As the resistivity in the TAFF regime may be expressed as

$$
\rho(T, H)=\rho_{0} \exp \left(-U_{0}(T, H) / k_{\mathrm{B}} T\right),
$$

the activation energy, $U_{0}$, can be obtained by plotting the $\ln \left(\rho / \rho_{0}\right) v s .1 / T$. The graphs for the samples of Y-358 and YCa-358 are given in figure $3 \mathrm{a}$ and $\mathrm{b}$, respectively. From the figures, it is clear that the broadening for $\mathrm{YCa}-$ 358 is apparently reduced as compared to that of $\mathrm{Y}-358$, as expected. It is obvious that the resistive broadening caused by an applied magnetic field manifests itself with

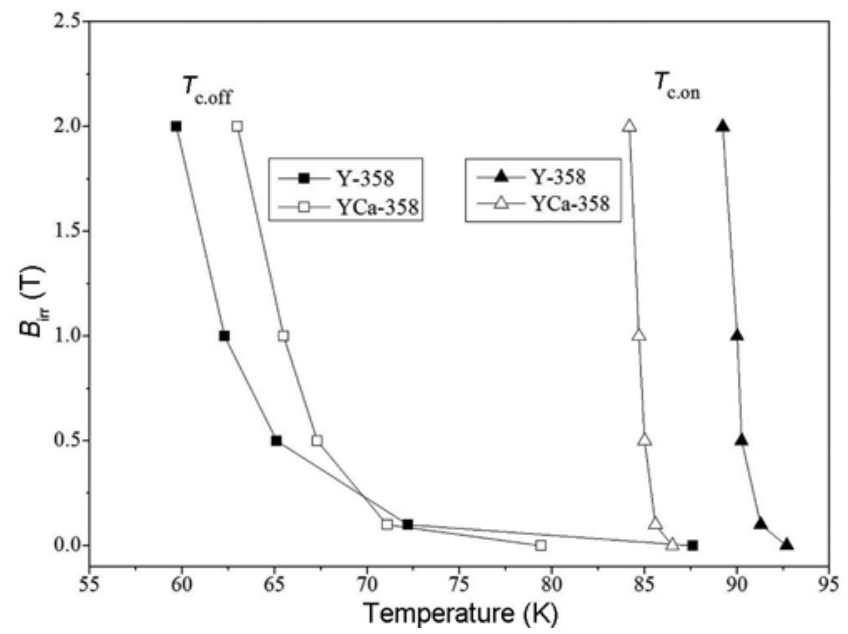

Figure 2. A comparison and variation of the irreversibility magnetic fields against the $T_{\text {c.on }}$ and $T_{\text {c.off }}$ of the compounds $\mathrm{Y}_{3} \mathrm{Ba}_{5} \mathrm{Cu}_{8} \mathrm{O}_{18}$ and $\mathrm{Y}_{3} \mathrm{Ba}_{5} \mathrm{Ca}_{2} \mathrm{Cu}_{8} \mathrm{O}_{18}$.

the $T_{\mathrm{c}}$ shifts to the lower temperatures. As mentioned above, this well-known behaviour is attributed to the flux flow resistivity. It is well established that lowering the temperature increases the pinning strength and hence the pinning force increases as well. When the Lorentz force per unit volume, $F_{\mathrm{L}}$, exceeds the pinning force per unit volume, $F_{\mathrm{P}}$, a flux flow arises and an electrical resistivity appears due to the flux flow. Hence, a higher magnetic field is needed to depinning the flux lines at lowest temperatures. As we have also mentioned earlier, this results in a shift of the zero resistivity temperature downwards as the magnetic field increases.

The other result, which can be deduced from the semilogarithmic Arrhenius plots of figure $3 a$ and $b$, is that indeed there is nearly an exponential dependence of $\rho$ on $1 / T$, for $T \ll T_{\text {c.on }}$, indicating that in the TAFF region the energy dissipation is due to the thermal activation of fluxes across the pinning barriers. Therefore, $U$-values can be obtained from the slopes of the nearly straight line portions of the curves at lower temperatures. In order to see the magnetic field dependence of the thermally activated flux flow activation energies, $U$, for the samples of 
Y-358 and YCa-358, they are plotted as a function of applied magnetic fields as shown in figure 4. It is obvious that when the magnetic field is gradually increased from
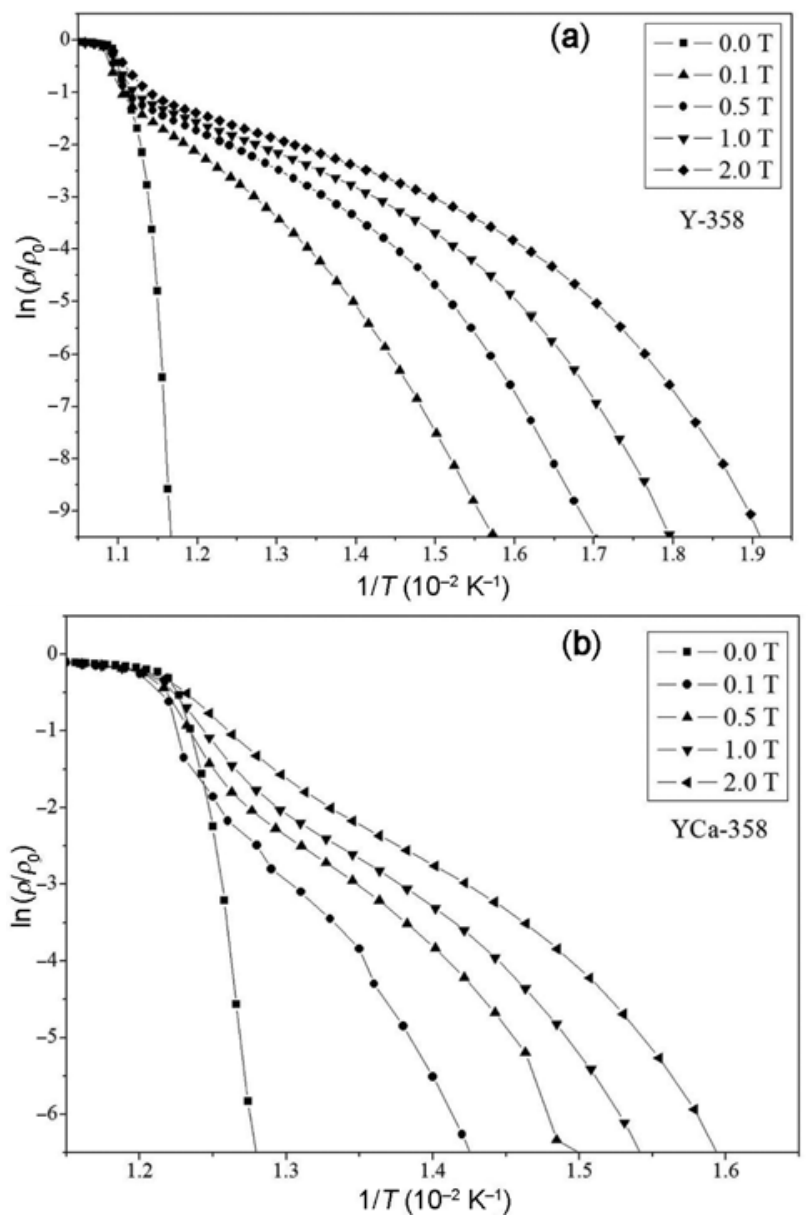

Figure 3. Arrhenius plot of the resistivity of the compounds (a) $\mathrm{Y}_{3} \mathrm{Ba}_{5} \mathrm{Cu}_{8} \mathrm{O}_{18}$ and (b) $\mathrm{Y}_{3} \mathrm{Ba}_{5} \mathrm{Ca}_{2} \mathrm{Cu}_{8} \mathrm{O}_{18}$

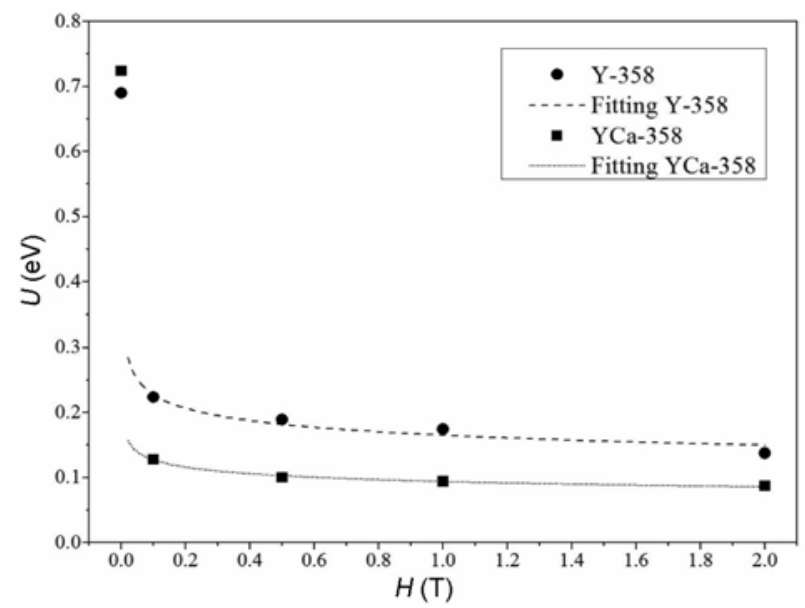

Figure 4. The magnetic field dependence of the activation energy, $U_{0}$, for the compounds $\mathrm{Y}_{3} \mathrm{Ba}_{5} \mathrm{Cu}_{8} \mathrm{O}_{18}$ and $\mathrm{Y}_{3} \mathrm{Ba}_{5} \mathrm{Ca}_{2}$ $\mathrm{Cu}_{8} \mathrm{O}_{18}$. Dotted lines are the theoretical fit of $U_{0}=c \cdot H^{-\alpha(H)}$ to the data.
0 to $2 \mathrm{~T}$, the rate of decline in pinning energy, $U$, decreases. Figure 4 also shows that $U(H)$ of $\mathrm{YCa}-358$ is lower than that of $\mathrm{Y}-358$ at the same field values. The activation energy reduces with the increase in the applied magnetic field, as well as doping $\mathrm{Ca}$ into the $\mathrm{Y}_{3} \mathrm{Ba}_{5} \mathrm{Cu}_{8} \mathrm{O}_{18}$. The $U$-values are found as 0.138 and $0.087 \mathrm{eV}$ in the Y-358 and YCa-358 samples under $2 \mathrm{~T}$ magnetic field, respectively. This confirms the destruction of weak link in the intergranular coupling the contraction of distance between the pinning centres and decrement of the energy barriers in the materials. It is attributed the decrease of flux pinning energy to a local suppression of the superconducting order parameter in the vicinity of $\mathrm{Ca}$ ions. ${ }^{17}$ Under magnetic fields below $\sim 0.2 \mathrm{~T}$, the pinning energy strongly depends on the value of the magnetic field. This indicates that below $\sim 0.2 \mathrm{~T}$, the applied magnetic field can penetrate only into the intergranular media. On the other hand, a possible interpretation for the differences between the two curves can be ascribed to the existence of different superconducting levels within the samples at the intergranular regions. ${ }^{18-20}$ Figure 4 also shows that the data quite well fit the power law equation

$$
U=c \cdot H^{-\alpha(H)}
$$

where $\alpha(H)$ is a function of field and $c$ a constant. Thus, from the curve fitting, it is revealed that for both of the compounds $U$ obeys the power law behaviour with respect to the applied magnetic field, but has slightly different exponents. The value of $\alpha$ depends on the orientation of the magnetic field with respect to the basal plane and the range of magnetic field. ${ }^{19}$ Table 2 shows the values of $c$ and $\alpha$ obtained from the fitting of the data of figure 4 to equation (2). Comparing the $c$ and $\alpha$ values it can be concluded that while the values of $\alpha$ increase those of $c$ decrease with $\mathrm{Ca}$ doping into the YBCO compound. As $\alpha$ is small and so that $U$ is almost field independent above $0.2 \mathrm{~T}$, one may also conclude that both of the compounds exhibit flux flow and impartial flux pinning. The slight difference in the values of $\alpha$ might be related to be due to the sensitivity of the pinning energy based on crystal structure characteristics such as oxygen contents, the real position of atoms, the quality of weak links, etc. Thus, the observed slight difference of $\alpha$ might be associated with the influence of microstructural defects. A comparison of X-ray diffraction (XRD) patterns of the samples of $\mathrm{Y}-358$ and $\mathrm{YCa}-358$ are represented in figure 5. The structure of the sample of Y-358 was determined to be predominantly monophasic with Pmmm47 symmetry, according to the powdered XRD analysis; however, small impurity peaks are observed, marked by asterisks, on the spectrum. It is assumed that some $\mathrm{CaCO}_{3}$ remains as an impurity in the $\mathrm{YCa}-358$ as seen in figure 5. The lattice parameters are obtained from the refinement process and found to be $a=3.88 \AA$, $b=3.84 \AA$ and $c=31.71 \AA$. Least-squares fit to the Pmmm47 space group shows a systematic decrease of the 


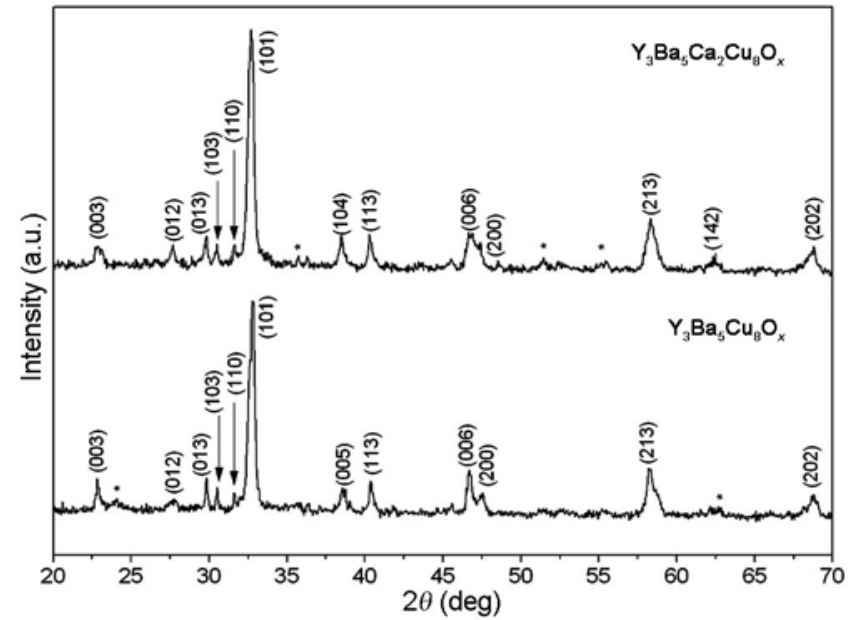

Figure 5. XRD pattern of the compounds $\mathrm{Y}_{3} \mathrm{Ba}_{5} \mathrm{Cu}_{8} \mathrm{O}_{18}$ and $\mathrm{Y}_{3} \mathrm{Ba}_{5} \mathrm{Ca}_{2} \mathrm{Cu}_{8} \mathrm{O}_{18}$.

orthorhombic distortion and a slight increase of the lattice parameters $a, c$ with the doping of Ca, i.e., $a=3.90 \AA$, $b=3.84 \AA$ and $c=31.74 \AA$ in the sample of YCa-358. The small increase in the lattice parameters may be due to the fact that $\mathrm{Ca}^{2+}$ has a bigger ionic radius than that of $\mathrm{Y}^{3+}$ (ionic radii; Y: $1.01 \AA$, Ba: $1.35 \AA, \mathrm{Cu}: 0.47 \AA$, O: $1.35 \AA$ and $\mathrm{Ca}: 1.34 \AA$ ). Furthermore, electrons may be introduced into the antibonding $\mathrm{Cu}-\mathrm{O}$ orbitals on the $a-b$ plane by the doping that tends to expand the $a$-dimension and suggest a strong disorder in the $a-b$ plane. These results may be attributed to the existence of much more disorder due to a greater number of $\mathrm{Cu}$ sites to be doped by $\mathrm{Ca}$ in Y-358 to Y-123. By comparing the XRD pattern of the $\mathrm{Y}-358$ with that of $\mathrm{Y}-123$, it is seen that the main peaks are the ones which exist in Y-123 except for the presence of some additional reflexions with low intensities. The lattice parameters of free added Y-358 are in agreement with Akhavan's report; ${ }^{7}$ the $a$ and $b$ parameters are very close to those of Y-123 (ref. 4) and the $c$ parameter of $\mathrm{Y}-358$ is almost three times to that of Y-123.

The temperature and field dependence of the $-T \ln \left(\rho / \rho_{0}\right)$ data for the samples of Y-358 and YCa-358 are shown in figure $6 \mathrm{a}$ and $\mathrm{b}$. In the field range $0.0 \leq H \leq 2.0 \mathrm{~T}$ and below $T_{\mathrm{c}}$, the data are clearly temperature and field dependent. As it can be seen the data first increase then decrease, forming a kind of peak (kink) with the decrease in temperature. In addition, the peaks shift to the lower temperatures with the increase in field. Furthermore, the magnitudes of $-T \ln \left(\rho / \rho_{0}\right)$ curves (roughly the values of $U$ ) monotonously decrease with the increase in the magnetic field, as also demonstrated in figure 4. In addition, well below the mentioned peak type of maxima the data seem to be field independent and attain linear temperature dependence. This region corresponds to the low resistivity portion of the Arrhenius curves depicted in figure $3 a$ and $b$, i.e., the regime where vortex motion is determined by
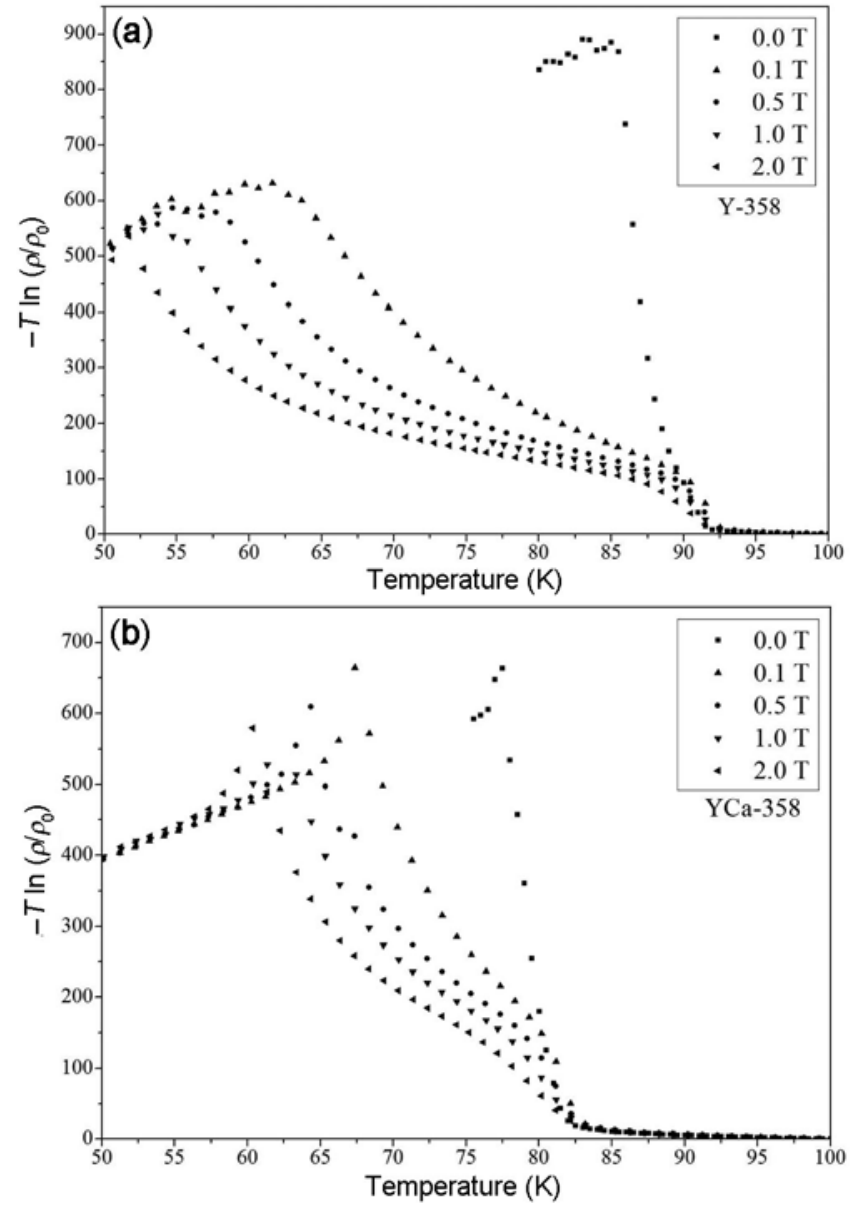

Figure 6. Experimental $-T \ln \left(\rho / \rho_{0}\right)$ data against temperature for the samples of (a) $\mathrm{Y}_{3} \mathrm{Ba}_{5} \mathrm{Cu}_{8} \mathrm{O}_{18}$ and (b) $\mathrm{Y}_{3} \mathrm{Ba}_{5} \mathrm{Ca}_{2} \mathrm{Cu}_{8} \mathrm{O}_{18}$.

the TAFF. The appearance of peaks has been explained by the possibility of showing either transition from flux flow/creep to TAFF region or the crossover from two-dimensional (2D) to three-dimensional (3D) vortex nature. $^{18}$

The upper critical fields, $H_{\mathrm{c} 2}$, of the compounds were found by using the $\rho=\rho_{\mathrm{N}}(90 \%)$ relation, where $\rho_{\mathrm{Ns}}$ are the normal state resistivities at the critical temperatures $T_{\text {c.on}}$, related to each field value. ${ }^{20}$ The calculated upper critical fields, $H_{\mathrm{c} 2}$, vs. the critical temperatures are plotted in figure 7. As it can be seen from the figure, the temperature dependence of $H_{\mathrm{c} 2}$ for both of the compounds exhibit similar behaviours. However, as expected, the curve for YCa-358 is shifted to lower temperatures as compared to that of $\mathrm{Y}-358$. As, at low temperatures, experimentally finding the $H_{\mathrm{c} 2}$ is not possible, in order to see the behaviours of the upper critical fields at temperatures close to zero, we have used a theoretical model defined by the Werthamer-Helfand-Hohenberg (WHH) formula ${ }^{21}$

$$
H_{\mathrm{c} 2}(0)=0.69 T_{\mathrm{c}}\left[\frac{\mathrm{d} H_{c 2}(T)}{\mathrm{d} T}\right]_{T_{\mathrm{c}}},
$$


where the $\left(\mathrm{d} H_{\mathrm{c} 2}(T) / \mathrm{d} T\right) \mathrm{s}$ are determined using the slopes at $T_{\mathrm{c}}$ of the curves in figure 7 . The values of calculated upper critical fields at zero temperature for the compounds Y-358 and YCa-358 are tabulated in table 2. Those are quite acceptable values for a good type-II superconductor. It is found that the $H_{\mathrm{c} 2}(0)$ decreases from 40.65 to $38.18 \mathrm{~T}$ with the doping of $\mathrm{Ca}$ into the $\mathrm{Y}-358$ compound because of the pair-breaking mechanism. This shows that the addition of $\mathrm{Ca}$ deteriorates superconducting properties, which is also consistent with XRD measurements. This result is also compatible with our other results, such as, the decrease of the critical temperature, the critical current density and the superconducting volume fraction with the addition of $\mathrm{Ca}$, reported in our previous study. ${ }^{9}$ We can also point out that due to the doping of $\mathrm{Ca}$ into Y-358, the flux pinning centres are negatively affected, as the decrease in the upper critical field value as well as the decrease in $U$. This conclusion is also verified by the increase in the $T_{\text {c.off }}$ values with the addition of $\mathrm{Ca}$ to Y-358 (see table 1 for high applied fields).

One of the most important parameters in the superconductivity is the coherence length, $\xi$. In this work, it is calculated by using the Ginzburg-Landau relation

$$
H_{\mathrm{c} 2}(0)=\frac{\Phi_{0}}{2 \pi \xi^{2}(0)},
$$

where $\Phi_{0}$ is the quanta of flux $\left(2.07 \times 10^{-15} \mathrm{~T} \mathrm{~m}^{2}\right), H_{\mathrm{c} 2}(0)$ is the upper critical field at zero temperature. ${ }^{22}$ The

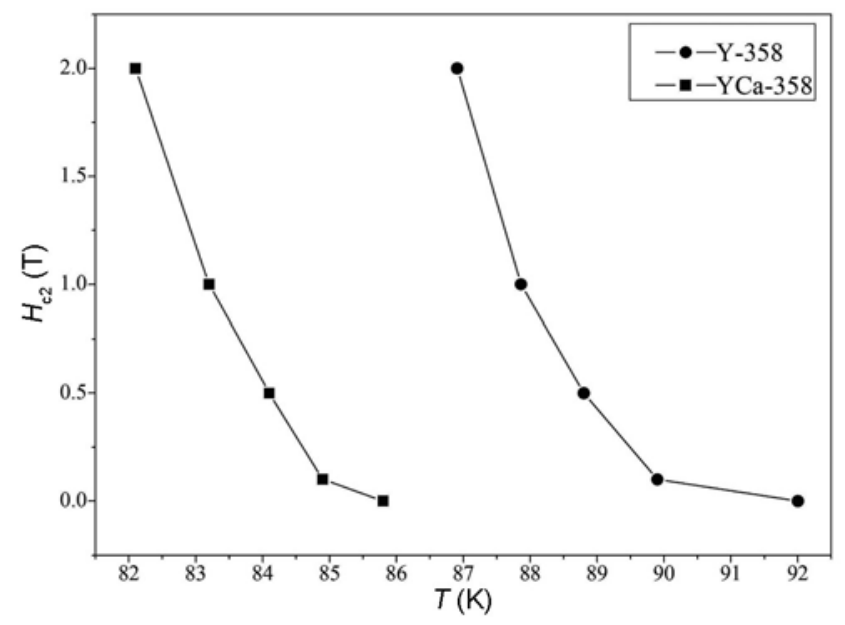

Figure 7. Upper critical fields $H_{\mathrm{c} 2}(T)$ as a function of temperature for the samples of $\mathrm{Y}_{3} \mathrm{Ba}_{5} \mathrm{Cu}_{8} \mathrm{O}_{18}$ and $\mathrm{Y}_{3} \mathrm{Ba}_{5} \mathrm{Ca}_{2} \mathrm{Cu}_{8} \mathrm{O}_{18}$. calculated values of the coherence length at zero temperature are tabulated in table 2 . The $\xi_{(0)}$-values increase with the addition $\mathrm{Ca}$ to the compound $\mathrm{Y}-358$ from 28.47 to $29.38 \AA$, presenting that the doped Ca ions play an important role on the pair-breaking mechanism in the Y-358 system. In fact, doping of $\mathrm{Ca}$ ions with a comparable ionic radius of $\mathrm{Y}, \mathrm{Ba}, \mathrm{Cu}$ and $\mathrm{O}$ may increase the hole concentration in Y-358 system, and so doped $\mathrm{Ca}$ might supply the mobile holes into $\mathrm{CuO}_{2}$ planes and increases hole carrier concentration per plane. It seems also that the depression of $T_{\mathrm{c}}$ with the addition of $\mathrm{Ca}$ may be either due to the decrease in oxygen content in $\mathrm{CuO}$ chains, or due to the trapping of mobile holes or other mechanism connected with oxygen vacancy disorder for others. ${ }^{23-25}$ Therefore, in general, the above results indicate that doping of $\mathrm{Ca}$ into the compound $\mathrm{Y}-358$ causes a negative effect in its superconducting properties.

\section{Conclusion}

In summary, we have investigated the effects of the applied magnetic fields on the superconducting properties of the superconductor $\mathrm{Y}_{3} \mathrm{Ba}_{5} \mathrm{Cu}_{8} \mathrm{O}_{18}$ with the addition of $\mathrm{Ca}$. The important physical properties of the compounds, Y-358 and YCa-358, such as $T_{\mathrm{c}}, U, B_{\text {irr }}, H_{\mathrm{c} 2}, \xi_{(0)}$ values are inferred from the $\rho-T$ curves under DC magnetic fields up to $2 \mathrm{~T}$. The temperature dependence of the resistivity values well below $T_{\text {c.on }}$ can be described by a TAFF model. Namely, the resistivities in the TAFF regime of the samples of Y-358 and YCa-358 under magnetic fields can be described by the TAFF resistivity formula

$$
\rho(H, T)=\rho_{0} \exp \left(-U(T, H) / k_{\mathrm{B}} T\right),
$$

where a power law dependence of $U$ on magnetic field of the form $U \sim H^{-\alpha}$ has been observed. The $\alpha$-value obtained for Y-358 sample is less than that for YCa-358 sample, showing that the sensitivity of $\mathrm{Y}-358$ with magnetic field is also less than that of YCa-358. The superconducting transition temperatures, $T_{\text {c.on }}$, decrease whereas $T_{\text {c.off }}$ increases with the addition of $\mathrm{Ca}$ into the $\mathrm{Y}_{3} \mathrm{Ba}_{5} \mathrm{Cu}_{8} \mathrm{O}_{18}$ superconductor in the increasing applied magnetic fields. The electric resistivity shows a strong sensitivity with applied magnetic fields and it is related to the considerably weaker connections between the superconducting grains in the Y-358 system. In addition, the activation energy, $U$, is also found to decrease with the addition of $\mathrm{Ca}$ into

Table 2. The $H_{\mathrm{c} 2}, \xi, \alpha$ and $c$ parameters of the compounds Y-358 and YCa-358 defined in equations (3)-(5).

\begin{tabular}{lcccc}
\hline & \multicolumn{2}{l}{ Values of parameters } & Upper critical field \\
\cline { 2 - 3 } Sample code & $\alpha$ & $c$ & & $\begin{array}{c}\text { The coherence length }(\AA) \\
\xi(0)\end{array}$ \\
\hline Y-358 & 0.13 & 0.165 & 40.7 & 28.5 \\
YCa-358 & 0.14 & 0.094 & 38.2 & 29.4 \\
\hline
\end{tabular}


the compound Y-358, but its magnetic field dependence does not change. Furthermore, the $H_{\mathrm{c} 2}(0)$-value decreases, whereas the coherence length, $\xi_{(0)}$, increases with the doping of $\mathrm{Ca}$ into $\mathrm{Y}-358$. As the coherence length is related to the vortex size, non-superconducting regions increase with $\mathrm{Ca}$ addition. All of these results are compatible with our previous observations, such as the decrease of the critical current density and flux pinning force with the addition of $\mathrm{Ca}$ into the compound $\mathrm{Y}-358 .{ }^{9}$

\section{Acknowledgements}

We thank Deniz Çetin for his contribution to language check. This work is supported by the Research Fund of Çukurova University, Adana, Turkey, under grant contracts FEF2009YL30, FEF2013BAP16 and FEF2013BAP29.

\section{References}

1. Wuyts B, Moshchalkov V and Bruynseraede Y 1996 Phys. Rev. B 539418

2. Minami $\mathrm{H}$ et al 1995 Advances in superconductivity VII (Berlin: Springer) p 141

3. Minami H and Uwe H 1997 Physica C 282-287 1193

4. Wu M K, Ashburn J R, Tornk C J, Hor P H, Meng R L, Gao L, Huang Z J, Wang Y Q and Chu C W 1987 Phys. Rev. Lett. $\mathbf{5 8} 908$

5. Marsh P, Fleming R M, Mandich M L, DeSantolo A M, Kwo J, Hong M and Martinez-Miranda L J 1988 Nature 336660

6. Bordet P, Chaillout C, Chenavas J, Hodeau J L, Marezio M, Karpinski J and Kaldis E 1988 Nature 334596

7. Aliabadi A, Akhavan-Farshchi Y and Akhavan M 2009 Physica C $\mathbf{4 6 9} 2012$
8. Ayas A O, Ekicibil A, Cetin S K, Coskun A, Er A O, Ufuktepe Y, Firat T and Kiymac K 2011 J. Supercond. Nov. Magn. 242243

9. Ekicibil A, Kilic Cetin S, Ayas A O, Coskun A, Firat T and Kiymac K 2011 Solid State Sci. 131954

10. Yazici D, Erdem M and Ozcelik B 2012 J. Supercond. Nov. Magn. 251811

11. Yang H C, Wang L M and Horng H E 1999 Phys. Rev. B 5913

12. Hughes D 1988 Cryogenics 28674

13. Palstra T T M, Batlogg B, van Dover R B, Schneemeyer L F and Wasczak J V 1990 Phys. Rev. B 416621

14. Kucera J T, Orlando T P, Virshup G and Eckstein J N 1992 Phys. Rev. B 4611004

15. Wagner P, Hillmer F, Frey U and Adrian H 1994 Phys. Rev. B 4913184

16. Zhang Y Z, Wang Z, Lu X F and Wen H H 2005 Phys. Rev. B 71052502

17. Abov-Aly A I, Mahmoud S A, Awad R and Barakat M M E 2010 J. Supercond. Nov. Magn. 23157

18. Govea-Alcaide E, Garcia-Fornaris J, Mune P and Jardim R F 2007 Eur. Phys. J. B $\mathbf{5 8} 373$

19. Sharma D, Kumar R and Awana V P S 2012 Solid State Commun. 152941

20. Erdem M, Ozturk O, Yucel E, Altintas S P, Varilci A, Terzioglu C and Belenli I 2011 Physica B 406709

21. Moodera J S, Meservey R, Tkaczyk J E, Hao C X, Gibson G A and Tedrow P M 1988 Phys. Rev. B 37619

22. Oh B et al 1988 Phys. Rev. B 377861

23. Awana V P S and Narlikar A V 1994 Phys. Rev. B 49 6353

24. Awana V P S, Malik S K, Yelon W B, Cardoso C A, de Lima O F, Gupta A, Sedky A, Samanta S B and Narlikar A V 2000 Physica C 338197

25. Manthiram A, Lee S J and Goodenough J B 1988 J. Solid State Chem. $\mathbf{7 3} 278$ 